\title{
PROKLA-Redaktion
}

\section{Editorial: Familie und Staat}

„Wer sich in Familie begibt, kommt darin um" - diese bittere Diagnose des Wiener Schriftstellers Heimito von Doderer, der seit den 1950er Jahren mit Romanen wie Die Strudlhofstiege oder Die Dämonen bekannt wurde, hätte die Mehrzahl seiner bürgerlichen Leser/innen wohl kaum geteilt, galt doch nicht nur ihnen, sondern sämtlichen gesellschaftlichen Schichten die Familie als einzig erstrebenswerte Lebensform. Zwar warfen auch andere Schriftsteller wie Arthur Schnitzler oder Thomas Mann einen Blick in die seelischen Abgründe, die sich in Familien auftun konnten, und schilderten beredt die möglichen psychischen Beschädigungen, die vor allem Frauen und Kinder, aber keineswegs nur sie, trafen. Auch Sozialwissenschaftler wie Max Horkheimer oder Erich Fromm stellten bereits 1936 in den Studien über Autorität und Familie kritische Überlegungen zur Entstehung autoritärer Persönlichkeiten an. Die patriarchale Kleinfamilie, in der sämtliche Mitglieder in ihrer Abhängigkeit von dem männlichen Ernährer standen, wurde als eine der tieferen Ursachen dafür gesehen, dass Kinder heranwuchsen, die unselbständig blieben, von Minderwertigkeitsgefühlen geplagt waren und sich bereitwillig unterordneten.

Jahrzehnte später wurden diese Erkenntnisse in der Studentenbewegung wieder aufgenommen und ausgiebig in Hochschul-Seminaren diskutiert. Eine beherzte Minderheit versuchte auch umgehend, sie in die Tat umzusetzen. Indem Kommunen und Kinderläden gegründet wurden, sollten die bisherigen unterdrückenden Strukturen aufgehoben werden. In Kommunen wurden tendenziell alle Prinzipien der bürgerlichen Familie privates Eigentum, sexuelle Monogamie, interne Hierarchie - auf den Kopf gestellt, was viele der damals Beteiligten im Rückblick inzwischen als recht zwiespältige, gelegentlich sogar traumatische Erfahrung erinnern. Dagegen haben die Experimente mit neuen Erziehungsvorstellungen in den Kinderläden, anfangs in der Öffentlichkeit ängstlich beäugt und unter den Verdacht des Zusammenbruchs aller Werte gestellt, längerfristig positive Auswirkungen auf die gängigen Konzepte von Kindergärten gehabt. Kinder sollten nun nicht mehr in erster Linie diszipliniert werden, zu festgesetzten Zeiten schlafen und alle die gleichen possierlichen Wichtelmännchen zusammen bauen, sondern ihre Kreativität entfalten, sich selbst und andere als selbständig erfahren und frei spielen, wobei traditionelle Reformkonzepte wie solche der Montessori-Kindergärten wieder zu neuem Ansehen kamen.

Die Familie als auf Dauer angelegte Lebensgemeinschaft von Eltern und Kindern hat in den letzten Jahrzehnten als gesellschaftlicher Leitstern kaum an Strahlkraft eingebüßt. Trotzdem die Zahl der Eheschließungen seit Jahren tendenziell zurückgegangen ist und die Scheidungen 
zugenommen haben, leben heutzutage rund drei Viertel aller minderjährigen Kinder in Familien mit ihren verheirateten Eltern. Auch in der literarischen Öffentlichkeit ist das Thema Familie - besonders gern als Betrachtung mehrerer Generationen - in den letzten Jahren unübersehbar dominant geworden. So ging der Deutsche Buchpreis seit 2005 fast in jedem Jahr an einen Familienroman, 2005 an Arno Geiger für Uns geht es gut, 2006 an Katharina Hacker für Die Habenichtse, 2007 an Julia Franck für Die Mittagsfrau, 2008 an Uwe Tellkamp für Der Turm, 2010 an Melinda Nadj Abouji für Tauben fliegen auf, 2011 an Eugen Ruge für In Zeiten abnehmenden Lichts.

Dies alles sagt jedoch wenig über die heutige gesellschaftliche Praxis und die Alltagsrealität von Familien aus, vor allem nichts darüber, in welchem politischen Rahmen sich diese entfalten. Vor diesem Hintergrund interessiert sich die PROKLA 173 vor allem für den Zusammenhang zwischen Familie und Staat: Inwiefern ist die Familie eine 'Staatsangelegenheit'? Wie sind die familienpolitischen Diskussionen und Reformen der vergangenen Jahre einzuschätzen? Inwiefern schreiben sich dabei Klasseninteressen und Umverteilungspolitiken in die Familienpolitik ein? Und: Wie und wo nehmen staatliche Politiken auch außerhalb der 'klassischen Familienpolitik' Einfluss auf die Rolle und die Formierung von Lebensgemeinschaften?

In einem einführenden Artikel setzt sich Katharina Hajek mit unterschiedlichen Debatten auseinander, die 'Familie' im Kontext moderner Staatlichkeit diskutieren. Dabei wird vor allem die soziale Konstruktion von Familie deutlich und die damit verbundene Trennung zwischen Öffentlich und Privat bzw. zwischen Politisch und Unpolitisch. Familie wird als ein Konzept und ein Gegenstand sichtbar, der sich überhaupt erst im Spannungsfeld verschiedenster Macht- und Herrschaftsverhältnisse konstituiert.

Deutlich wird allerdings auch, dass die (bürgerliche Klein-) Familie zwar enorm beständig, aber gerade auf Grund ihrer Verwobenheit mit dem modernen Staat durchaus wandelbar und anpassungsfähig ist. So legte der letzte Familienreport des Bundesministeriums für Familie, Senioren, Frauen und Jugend (BMFSFJ) ausführlich dar, dass in der bundesrepublikanischen Gesellschaft neben der traditionell-heterosexuellen Form von Familie (Mann, Frau, Kind/-er) inzwischen mannigfaltige Formen des Zusammenlebens entstanden sind. Und die Familienministerin der schwarz-gelben Koalition, Christina Schröder, ließ im Sommer 2013 verlauten: „Eine nachhaltige Familienpolitik respektiert die Vielfalt der Lebensentwürfe und unterstützt Menschen dabei, sich frei zu entscheiden, in welcher Weise sie füreinander Verantwortung übernehmen wollen." (www.bmfsff.de/BMSFSFJ/familie. $\operatorname{did}=20172 . h t m l)$

Im historischen Rückblick macht Christina Schröders Aufruf zu einer Art familienpolitischen Diversity-Politik aber auch klar, wie sehr sich der Diskurs in den vergangenen drei Generationen gewandelt hat. 1933 etwa verkündete Joseph Goebbels die politischen Ansprüche der Nationalsozialisten an die Familie mit den Worten: „Den ersten, besten und ihr gemäßesten Platz hat die Frau in der Familie und die wunderbarste Aufgabe, die sie erfüllen kann, ist die, ihrem Volk Kinder zu schenken" (zit. in Annette Kuhn (Hg.): Die Chronik der Frauen, Dortmund 1992, S. 477). An eigene Lebensentwürfe war hier nicht zu denken: Während die rassepolitisch erwünschten („arischen“) Frauen möglichst viele Kinder bekommen sollten, so die unerwünschten (nicht-,,arischen“" 
oder nicht „erbgesunden“) gar keine, was unter anderem mit Zwangssterilisierungen durchgesetzt wurde. Zwanzig Jahre später und sechzigJahre vor Ministerin Schröder vertrat ihr Amtsvorgänger Franz-Joseph Wuermeling andere, doch ebenso politisch von oben aufgestülpte Ansprüche, wenn er 1953 in einer katholischen Kirchenzeitung schrieb: „Millionen innerlich gesunder Familien mit rechtschaffen erzogenen Kindern sind als Sicherung gegen die drohende Gefahr der Völker des Ostens mindestens ebenso wichtig wie alle militärischen Sicherungen." (Kirchenzeitung des Erzbistums Köln, 6.12.1953, S. 780) Der westdeutschen Kleinfamilie war also die Aufgabe eines „Bollwerks gegen den Kommunismus" aufgetragen.

Der Vergleich dieser Zitate legt ein fundamentales Umdenken nahe. Nichts desto trotz gilt es zu fragen, inwieweit sich staatliche Setzungen, rechtliche Regelungen und die Transferleistungen für Familien heute tatsächlich am Leitbild der Selbstbestimmung und der freien Wahl von Formen des Zusammenlebens orientieren. Susanne Schultz setzt sich in ihrem Artikel mit der Rolle der Demographie in der Familienpolitik auseinander und stellt dabei fest: „Nachdem jahrzehntelang zumindest in der alten Bundesrepublik vor dem Hintergrund nationalsozialistischer Bevölkerungspolitik galt, den Umfang und die Zusammensetzung bzw. „Qualität“ der nationalen Bevölkerung nicht explizit zum Objekt familienpolitischer Bemühungen zu erklären, gerieten (in den 2000er Jahren) katastrophistisch aufgeladene Szenarien und Prognosen aus den aufblühenden Laboren der Demografie und Bevölkerungsstatistik ins strategische Zentrum der Debatte." Zwar lässt sich allein daraus sicher noch kein familienpolitischer backlash bzw. historische Kontinuität ableiten; deutlich wird jedoch, dass es gerade auch nach den Widersprüchen und Ungleichzeitigkeiten zwischen kulturellem und sozioökonomischem Wandel einerseits, zwischen Familienmodellen und Familienideologien andererseits zu fragen gilt, wenn das Verhältnis von Familie und Staat genauer bestimmt werden soll.

So steht beispielsweise das Ziel einer umfassenden Nutzung auch des Arbeitskräftepotentials von Frauen nicht selten im Konflikt mit dem staatlich geförderten und im Bewusstsein vieler Menschen fest verankerten Harmonieversprechen der bürgerlichen Kleinfamilie. Auch die deutsche Gleichstellungspolitik, die sich um die Vereinbarkeit von Beruf und Familie sorgt, verweist die Frauen mit dem Versprechen der Gleichberechtigung auf den kapitalistischen (Arbeits-)Markt. Audrey Podann hinterfragt in ihrem Beitrag diese einseitig arbeitsmarktorientierte Agenda heutiger Frauenpolitik und fordert eine Erneuerung feministischer Kritik an der Lohnarbeit.

Tomke König behandelt die „symbolische Geschlechterordnung" in ihren Veränderungen wie Beharrlichkeiten. Im Mittelpunkt ihrer Überlegungen stehen die Hausarbeit und jene Arrangements, in welchen Paare diese im Alltag verrichten. Dabei entwickelt sie die These einer ,paradoxalen Gleichzeitigkeit unterschiedlicher Ideale“. König stellt am Ende ihres Beitrags die Frage, wer sich eigentlich unter den vorherrschenden Bedingungen von Flexibilisierung und einer zunehmenden Prekarisierung wann und wie erholt. Für die weitere Diskussion schlägt sie schließlich vor, die Trennung von Beruflichem und Privatem im Kontext von Sozialpolitik als „sozialer Infrastruktur“ neu zu überdenken.

Nicht zu übersehen sind die unterschiedlichen Standards der bundesdeutschen Familienpolitik, je nachdem, um welche Familien es sich jeweils handelt: 
Für Familien, die ALG II beziehen, wird das Ziel der Familienförderung suspendiert und der „stumme Zwang der Verhältnisse“ über den Ausschluss vom Eltern- und Betreuungsgeld verschärft. Familienpolitik wird damit zum Medium der Exklusion bzw. zum funktionalen Korrelat einer Niedriglohnpolitik. Auch für Flüchtlinge gilt der hehre Anspruch, familiärer Zusammenhalt solle gefördert werden, häufig nicht, so wenn Eltern oder Kindern das Bleiberecht verweigert wird. Gerade an der Schnittstelle zwischen Migrations- und Familienpolitik werden, wie Anna Jüschke und Katharina Schoenes in ihrem Artikel zeigen, oft hetero-normative und traditonalistische Erwartungen an (transnationale) Partnerschaften herangetragen, die für biodeutsche Paare kaum mehr gelten. Deutlich wird daran auch noch einmal, dass 'die Familie' schlussendlich nicht nur im Kontext kapitalistischer Staatlichkeit untersucht und verstanden werden muss, sondern zudem auch unentwirrbar mit der Konstruktion von Rasse und Nation verwoben ist.

Volker Woltersdorff diskutiert die paradoxe Gleichzeitigkeit von Flexibilisierung und Persistenz der traditionellen Formen von Sexualität und Familie im Neoliberalismus. Obwohl die heteronormativen Formen von Geschlecht, Verwandtschaft und Familie zunehmend prekär werden, Diversität etwa in der Form der eingetragenen Partnerschaft für gleichgeschlechtliche Paare anerkannt wird, haben diese traditionellen Formen ihre normative Kraft noch längst nicht verloren und dienen weiterhin als „regulatorisches Ideal“ (Judith Butler). Heinz-Jürgen Voß wirft einen kritischen
Blick auf die bundesdeutsche Debatte um die 'Homo-Ehe' und setzt sich mit dem Wahlfamilien-Modell und dem französischen Pacte Civil de Solidarité (PACS) als Alternativen zur gleichgeschlechtlichen Ehe auseinander. Dass sich die 'Homo-Ehe' durchgesetzt hat, lag wohl nicht zuletzt daran, „weil sie am besten zur wichtigsten Institution passt, auf der normative Heterosexualität und moralischer Staat basieren: zur Ehe." (Nancy Peter Wagenknecht: Nur für gute Mädchen. Jungle world, Nr. 47, 15.11.2000).

Außerhalb des Schwerpunkts beschäftigt sich Errol Babacan mit den GeziPark Protesten in der Türkei, die sich im Juni 2012 zu einem Aufstand gegen die Zumutungen eines beschleunigten kapitalistischen Wachstums und ihres islamisch-konservativen Reproduktionsmodus entwickelten. Sowohl die unterschiedlichen Enteignungsprozesse, die dem neoliberalen Wachstumsmodell zugrunde liegen, als auch der islamistisch-konservative Kulturkampf erfuhren nun breiten Widerstand. Zwar könne in der Türkei noch nicht von einer Krise der neoliberalen Hegemonie gesprochen werden, doch werde eine Selbstermächtigung subalterner Klassen deutlich, die gemeinsames Lernen und oppositionelles Handeln ermögliche.

Es bleibt noch eine traurige Nachricht mitzuteilen. Am 17. August ist Thomas Hurtienne verstorben. Er war Autor und bis 1986 Redaktionsmitglied der PROKLA. Auch danach blieb er der PROKLA als Mitglied in der „Vereinigung zur Kritik der politischen Ökonomie“ verbunden. Urs Müller-Plantenberg erinnert in einem Nachruf an unseren Freund und Kollegen. 